\title{
EBIC and Luminescence Studies of Defects in Solar Cells
}

\author{
O. Breitenstein ${ }^{1}$, J. Bauer ${ }^{1}$, M. Kittler ${ }^{2}$, T. Arguirov ${ }^{2}$ and W. Seifert ${ }^{2}$ \\ ${ }^{1}$ Max Planck Institute of Microstructure Physics, Weinberg 2, Halle, Germany \\ ${ }^{2}$ IHP/BTU Joint Lab, Konrad-Wachsmann-Allee 1, Cottbus, Germany
}

\begin{abstract}
Summary: Electron beam-induced current (EBIC) can be used to detect electronic irregularities in solar cells, such as shunts and precipitates, and to perform physical characterization of defects by, e.g. measuring the temperature dependence of their recombination activity. Recently also luminescence methods such as electroluminescence (EL) and photoluminescence (PL) have been shown to provide useful information on crystal defects in solar cells. In this contribution it will be shown that the combined application of EBIC, EL and PL may deliver useful information on the presence and on the physical properties of crystal defects in silicon solar cells. Also pre-breakdown sites in multicrystalline cells can be investigated by reversebias EL and by microplasma-type EBIC, in comparison with lock-in thermography investigations. SCANNING 30: 331-338, 2008. (C) 2008 Wiley Periodicals, Inc.
\end{abstract}

Key words: EBIC, solar cells, luminescence, precipitates, pre-breakdownBMU0327650 B+D

\section{Introduction}

The scanning electron microscope (SEM) investigation of semiconductor devices in the current generation mode is called electron beaminduced current (EBIC) imaging (Holt and Joy 1989). This imaging mode, together with the usual secondary electron (SE) imaging mode, provides a number of unique possibilities to investigate defects

\footnotetext{
Address for reprints: O. Breitenstein, Max Planck Institute of Microstructure Physics, Weinberg 2, D-06120 Halle, Germany E-mail: breiten@mpi-halle.mpg.de

Received 17 April 2008; Accepted with revision 29 April 2008
}

DOI $10.1002 /$ sca.20112

Published online 16 June 2008 in Wiley InterScience (www.interscience. wiley.com) in semiconductor devices and materials and particularly in solar cells with high spatial resolution. There are several different sample geometries possible for EBIC. The standard EBIC geometry is a surface Schottky diode, which can be made on a polished clean Si surface by evaporating a thin layer of $\mathrm{Al}$ or $\mathrm{Ti}$ for $\mathrm{p}$-material or $\mathrm{Au}$ for $\mathrm{n}$-material. Although this geometry is often used for investigating defects in solar materials, it has some limitations. For example, the diffusion lengths in silicon solar cells are so large (typically several $100 \mu \mathrm{m})$ compared with the penetration depth of incident-beam electrons of only a few microns that diffusion-length-related phenomena cannot be investigated by EBIC. Moreover, because of the strong gettering effects of phosphorus diffusion and hydrogen passivation procedures, the recombination activity of individual dislocations is often very weak, so that they do not exhibit measurable EBIC contrast, at least not at room temperature. Therefore, low-temperature EBIC is a very important tool to investigate the electronic activity of defects in solar silicon. This technique may be combined with temperature-dependent luminescence investigations, which will be demonstrated in the first part of this contribution. In the second part the use of nonconventional sample geometries like "backside EBIC" will be shown to provide decisive information on the electronic activity of microscopic precipitates, which may be present in solar silicon material. Finally, it will be shown that pre-breakdown sites in solar cells can be localized not only by lock-in thermography (LIT) and reverse-bias EL but also by EBIC under avalanche conditions.

\section{Temperature Dependence of Defect Activity in Si as Observed by EBIC and Photoluminescence (PL)}

The recombination behavior of extended crystal defects in $\mathrm{Si}$ as measured by EBIC is found to depend on sample temperature $T$ and injection level/ beam current, see e.g. Holt (1996) and references 
therein. The temperature dependence of the EBIC contrast $c(T)$ analyzed under low injection conditions is mainly investigated. At first glance $c(T)$ yields information whether deep or shallow levels dominate the recombination activity of the defects, e.g. Kittler and Seifert (1993). This is illustrated in Figure 1. Figure 1(a) shows two types of $c(T)$
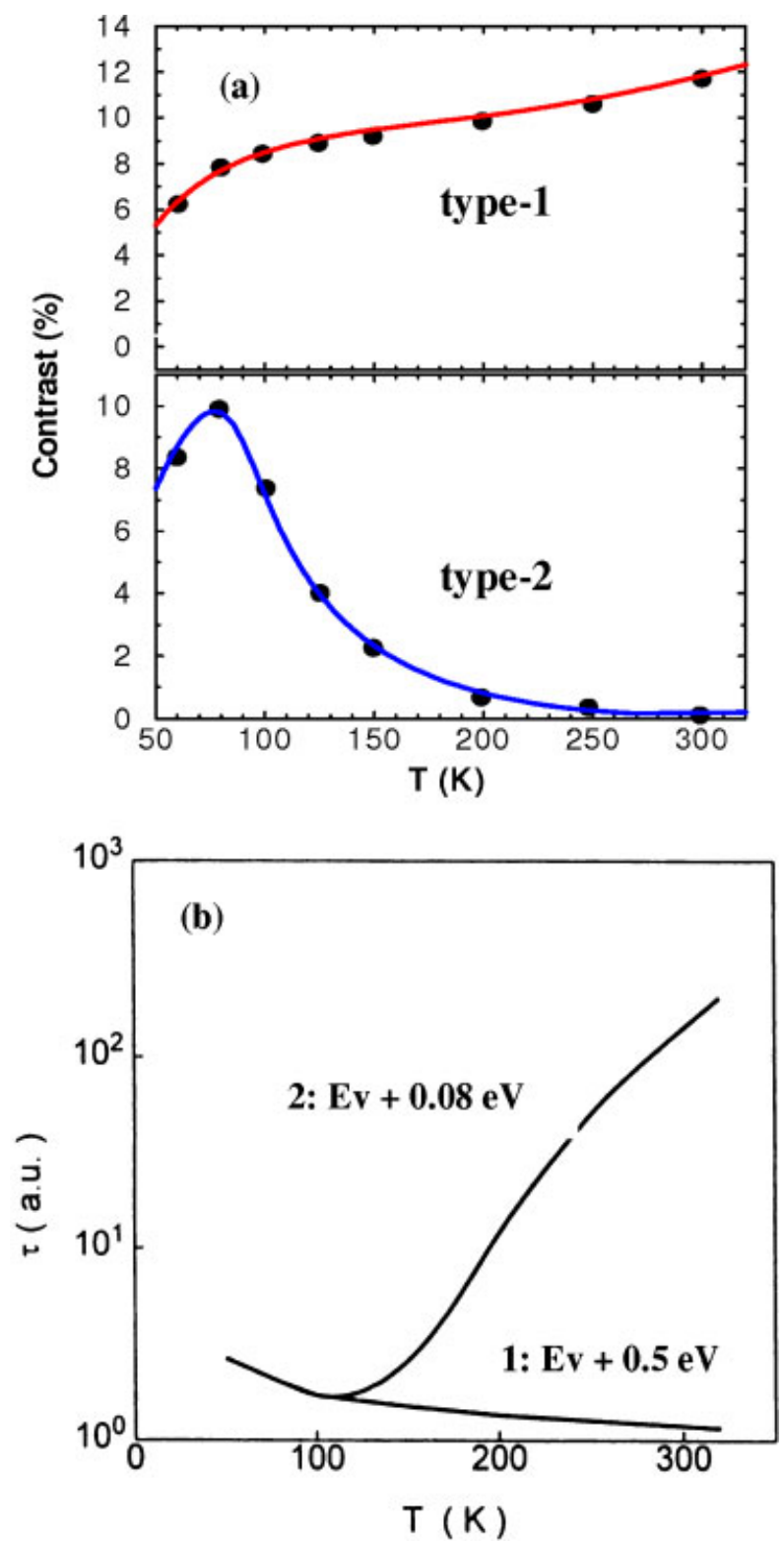

Fig 1. Temperature behavior of EBIC contrast $c(T)$ and lifetime $\tau(T)$. (a) Experimental $c(T)$ data measured for plastically deformed FZ-Si before (type-1) and after (type-2) phosphorus diffusion gettering. The solid lines are calculated using the model described in (Breitenstein and Langenkamp, 2003) yielding for the impurity concentration at the dislocations $N_{\mathrm{M}} 2 \times 10^{7}$ and $3 \times 10^{5} \mathrm{~cm}^{-1}$, respectively. (b) Normalized SRH lifetime $\tau$ calculated as a function of temperature $T$ under low injection conditions. Parameter is the trap position, which is $0.5 \mathrm{eV}$ above the valence band for curve 1 and $0.08 \mathrm{eV}$ above the valence band for curve 2, i.e. describing the behavior of deep and shallow levels, respectively. behavior of dislocations in $\mathrm{Si}$ observed experimentally, denoted type- 1 and type-2, respectively. The SRH lifetime calculated as a function of $T$ for deep and shallow levels, 0.5 or $0.08 \mathrm{eV}$ above the valance band, respectively, is shown in Figure 1(b). Taking into account that the contrast $c$ is inversely proportional to the lifetime at the defect $\tau_{\text {defect }}$, the type-1 behavior in Figure 1(a) can be related to deep levels, whereas type-2 behavior appears when shallow levels dominate the defect recombination.

Dislocations in $\mathrm{Si}$ are known to be connected with shallow levels about $80 \mathrm{meV}$ from the band edges, which are formed by the elastic strain field around the dislocations, e.g. Kveder et al. (2001). Deep levels at dislocations in Si may be caused by kinks or jogs, but are mainly formed by impurities/ metal atoms accommodated at the dislocations, e.g. Kittler et al. (1995). A model taking into account the recombination paths in the presence of both shallow and deep recombination levels at the dislocations (Kveder et al. 2001) allows to extract the density of deep levels/metal impurities $N_{\mathrm{M}}$ from the EBIC $c(T)$ behavior. Figure 2 shows the $c(T)$ behavior calculated with $N_{\mathrm{M}}$ as parameter. The type-1 contrast behavior depicted in Figure 1(a) was measured for dislocations produced by plastic deformation. Fitting the experimental data by the above model yields $N_{\mathrm{M}}=2 \times 10^{7} \mathrm{~cm}^{-1}$. Phosphorus diffusion gettering (PDG) of this sample changed the dislocation recombination behavior dramatically, giving rise to a type-2 $c(T)$ behavior. A value of $N_{\mathrm{M}}=3 \times 10^{5} \mathrm{~cm}^{-1}$ is obtained now by fitting the experimental data. Accordingly, external PDG cleans the dislocations and reduces the contamination by about two orders of magnitude. This example clearly demonstrates that EBIC is a powerful technique for in-depth analysis of the recombination behavior of defects in Si.

In the following we show that the temperature behavior of the luminescence in $\mathrm{Si}$ yields similar information. The big advantage of luminescence, as compared with EBIC, is the fact that it does not require contacts. Hence, it can be used as a fully nondestructive method. The prominent feature in Si luminescence is the band-to-band (BB) line at about $1.1 \mu \mathrm{m}$ emitted with the assistance of a TO phonon. The internal efficiency $\eta$ of the BB line calculated for $\mathrm{Si}$ at $300 \mathrm{~K}$ is shown in Figure 3 (for more details refer Kittler et al. 2005). SchockleyRead-Hall recombination dominates the internal efficiency for injection levels $\Delta n<10^{17} \mathrm{~cm}^{-3}$ while Auger recombination is the limiting process for $\Delta n>10^{17} \mathrm{~cm}^{-3}$. Accordingly, for $\Delta n<10^{17} \mathrm{~cm}^{-3}$ the intensity of the BB light escaping from the sample is reduced by recombination-active defects. Figure 4 shows EBIC and PL micrographs of the same multicrystalline (mc) Si sample. A focused light spot 


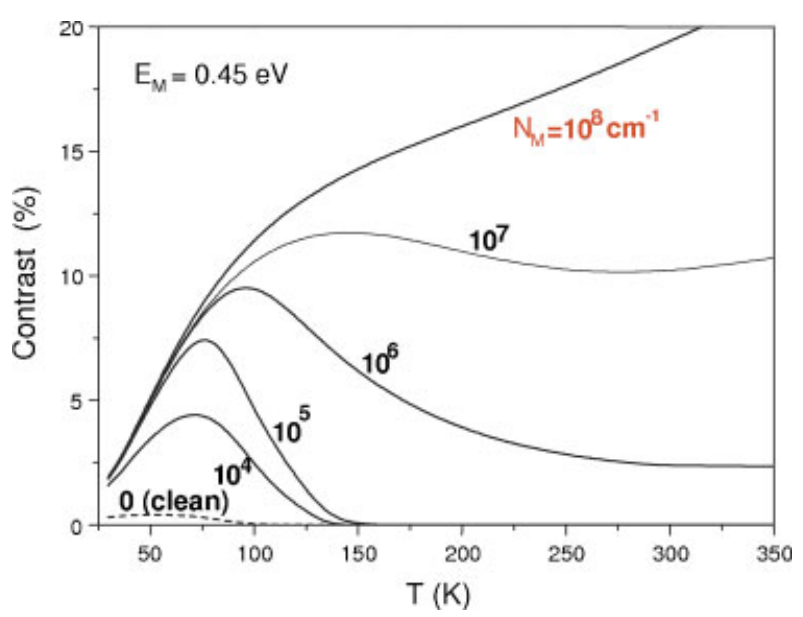

Fig 2. EBIC contrast behavior $c(T)$ for different concentrations $N_{\mathrm{M}}$ from 0 to $10^{8} \mathrm{~cm}^{-1}$ of deep level impurities at the dislocation, calculated by the model described in Breitenstein and Langenkamp (2003).

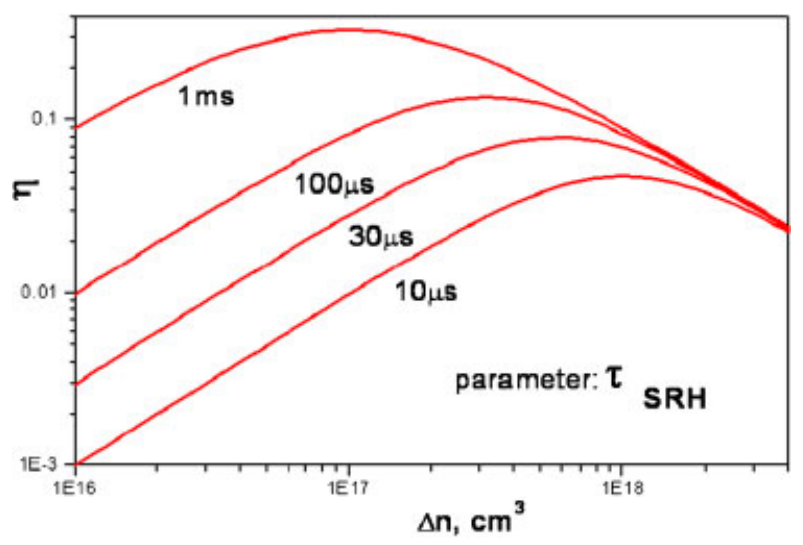

Fig 3. Internal quantum efficiency $\eta$ vs. excess carrier concentration $\Delta n$, calculated with SRH lifetime $\tau_{\mathrm{SRH}}$ as parameter. For details see Gaylord (1966).

was used for PL mapping to reach the same $\mu \mathrm{m}$ resolution as with EBIC.

In $\mathrm{Si}$ containing crystal dislocations also the quartet of $\mathrm{D}$ lines can be found in the luminescence spectrum, see e.g. Holt (1996) and references therein. The temperature behavior of the intensity of the $\mathrm{D}$ lines and the interrelation between D- and BBline intensities are not yet understood and still under discussion.

Here, we will focus on the temperature behavior of the $\mathrm{BB}$ line. In perfect monocrystalline $\mathrm{Si}$, the intensity of the BB line exhibits "normal" temperature behavior, i.e. it decreases when the temperature increases (Figure 5(a)). However, an opposite "anomalous" $T$ behavior can be observed in $\mathrm{Si}$, which contains a high density of crystal defects. Figure 5(b) shows the $T$ behavior of the BB line intensity, observed for $\mathrm{mc} \mathrm{Si}(\mathrm{mc}-\mathrm{Si})$ with a high defect density, in the as-grown state and after PDG.
In both cases the intensity increases with $T$, but the slope is considerably larger after PDG. Note that PDG was found by EBIC to clean the dislocations and to lead to a type-2 $c(T)$ behavior, which indicates that shallow dislocation levels dominate the recombination, see Figure 1(a). Owing to the dominant action of the shallow recombination levels, the lifetime $\tau_{\mathrm{SRH}}$ increases with increasing $T$ (Figure 1(b)). From Figure 3 it is evident that an increasing lifetime $\tau_{\mathrm{SRH}}$ causes an increase in the BB line efficiency/intensity. Hence, the observed "anomalous" temperature behavior of the BB light in the mc-Si sample is caused by dominance of shallow levels. The difference of the slopes shown in Figure 5(b) is because of a different level of contamination of the defects with deep impurities. A smaller slope corresponds to a higher contamination level at the defects.

\section{Investigation of Precipitates by "Backside EBIC"}

One of the advantages of the EBIC imaging mode is that it directly displays charge-collecting space charge regions, if they are connected with the current amplifier. In a solar cell usually only the space charge layer of the $\mathrm{p}-\mathrm{n}$ junction on the very top of the cell exists. However, in some mc cells a three-dimensional network of space charge regions can be found, which is due to grown-in $\mathrm{SiC}$ precipitates (Bauer et al. 2007b). These precipitates represent n-conducting filaments, which are crossing the whole cell and may result in an electrical short circuit (shunt) across the device. Such conducting channels can be investigated most effectively by mechanically removing the back contact, polishing and slightly etching the back face, and investigating the sample "face-down" by EBIC. For feeding out the current from the base, a rubbed-on $\mathrm{Ga}-\mathrm{In}$ contact on the base material is sufficient. The counter-electrode is the contacted emitter, which is lying down on the metallic sample holder. The "backside-EBIC" arrangement is schematically shown in Figure 6. Figure 7 shows typical results of such an investigation on a solar cell region, which was known to contain shunts. Before this investigation the surface was lightly etched in $\mathrm{HF}-\mathrm{HNO}_{3}$. As the collecting $\mathrm{p}-\mathrm{n}$ junction lies more than $150 \mu \mathrm{m}$ below the surface, and since the recombination velocity of the surface is very high, we actually do not expect significant carrier collection in this geometry. In this sample, however, in low magnification (Figure 7(a)) we see a bright line of high collection efficiency in EBIC. This can only be explained by the presence of a carrier-collecting region crossing the whole sample from bottom to top and feeding the minority carriers down to 
EBIC

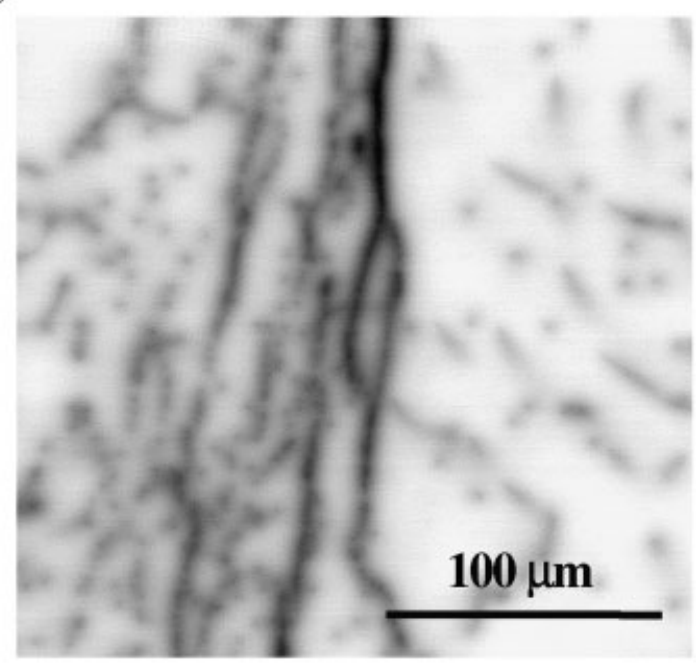

\section{PL (SiPHER by V. Higgs)}

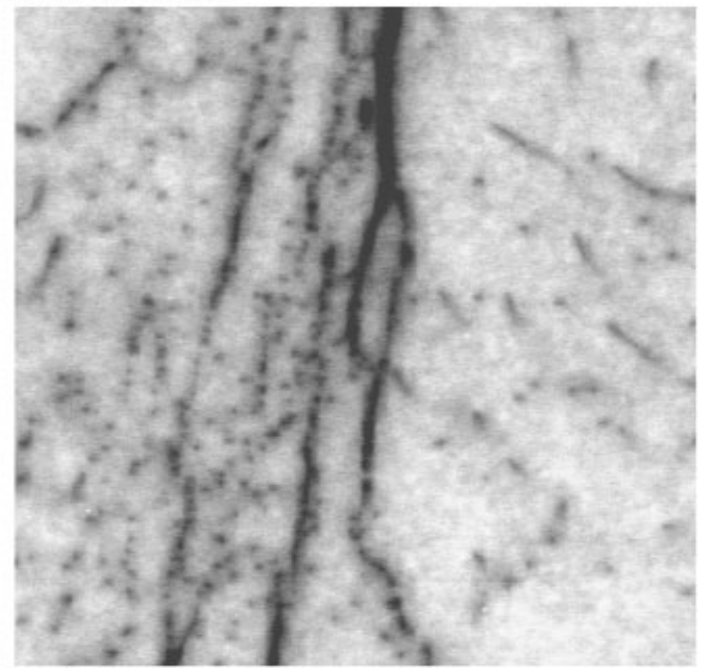

Fig 4. Comparison of EBIC ( $30 \mathrm{keV})$ and PL mapping $(532 \mathrm{~nm})$ on block cast mc-Si. PL mapping — using the BB line-was performed with a SiPHER ${ }^{\circledR}$ equipment by V. Higgs.

the $\mathrm{p}-\mathrm{n}$ junction. The SE image (Figure $7(\mathrm{~b})$ ) shows that this line is in the position of a grain boundary. The EBIC and SE images with higher magnification Figure 7(c) and (d) show that this bright EBIC line actually consists of single bright spots, which are carrier-collecting regions surrounding a row of filament-shaped precipitates lying in a grain boundary. Owing to the light etching, which did not affect the precipitates, they are somewhat sticking out of the surface. Energy-dispersive X-ray (EDX) investigations of such precipitates have revealed that they contain only $\mathrm{C}$ and $\mathrm{Si}$, and TEM investigations have proven that they consist of cubic (3C) SiC (Bauer et al. 2007a). In some cases also $\mathrm{Si}_{3} \mathrm{~N}_{4}$ filaments exist in grain boundaries, which, however, do not produce any carrier-conducting channels. Microscopic 4-point electrical measurements have shown that the $\mathrm{SiC}$ material is highly $\mathrm{n}$-conducting, but the $\mathrm{Si}_{3} \mathrm{~N}_{4}$ material is insulating (Bauer et al. 2007a). We believe that these $\mathrm{SiC}$ filaments are growing already in the block-casting process because of carbon contamination of the ingot and the environment. The n-type conductivity of the $\mathrm{SiC}$ material is most probably caused by nitrogen, which is the dominant shallow donor in SiC. The nitrogen stems from the silicon nitride coating of the quartz crucible, which gets slowly dissolved into the liquid Si. The presence 


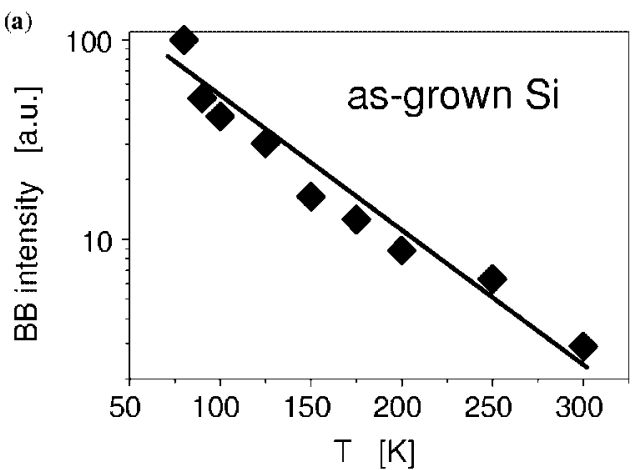

(b)

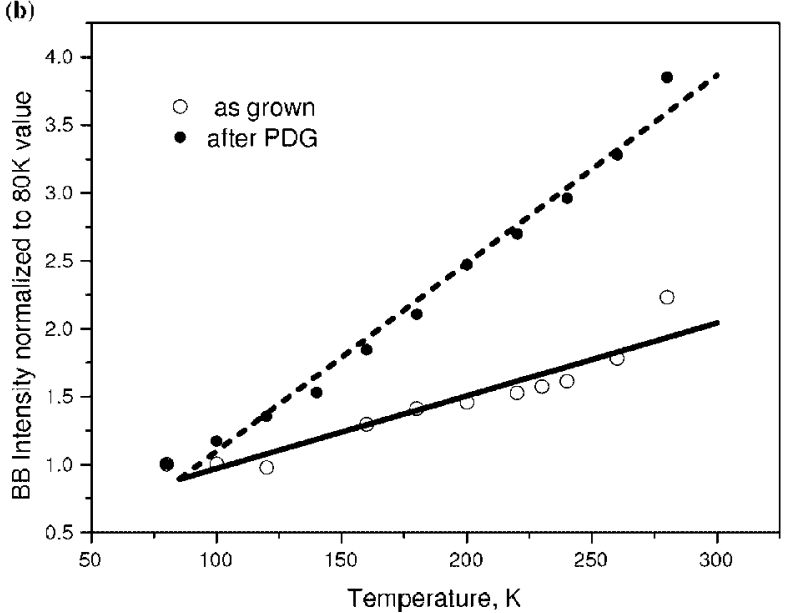

Fig 5. Temperature behavior of BB luminescence intensity. (a) Perfect as-grown $\mathrm{Si}$ exhibits an ordinary behavior, i.e. the intensity of the BB line decreases with increasing temperature. (b) Multicrystalline Si exhibits an anomalous behavior, i.e. the intensity increases with increasing temperature. The material in the as-grown state (open symbols) and after phosphorus diffusion gettering (full symbols) exhibits a different slope.

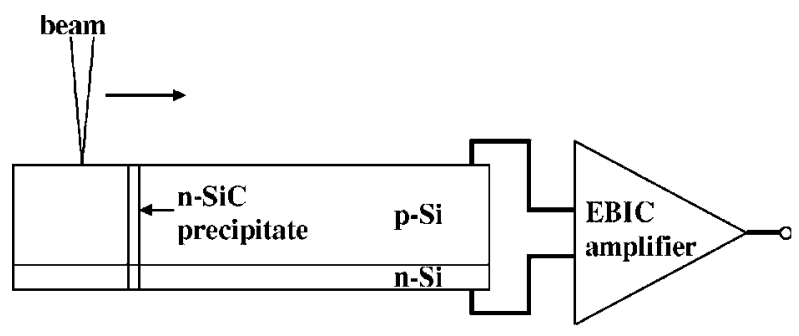

Fig 6. Backside EBIC arrangement scheme.

of high amounts of $\mathrm{N}$ in the $\mathrm{Si}$ melt is proven by the existence of $\mathrm{Si}_{3} \mathrm{~N}_{4}$ precipitates in this type of material (Bauer et al. 2007a,b).

\section{EBIC Investigation of Avalanche Processes}

It is well known that, under high reverse-bias, local breakdown may appear at sites of high electric field. There are two possible types of breakdown. Avalanche breakdown means that free carriers crossing the depletion region generate new electron-hole pairs by impact ionization. If the primary carriers are generated by the beam of an SEM working in EBIC mode, such multiplication sites are called "microplasma." It is well known that avalanche multiplication and microplasma formation may generate light (Gaylord 1966). The second breakdown type is internal field emission (IFE) or Zener breakdown. Here valence band electrons are tunneling through the energy gap to the conduction band, thereby also creating a free hole. In IFE no multiplication of photo- or electron beaminduced carriers occurs, hence this mechanism is not observable by EBIC. Under special circumstances (partial shading), solar cells in a module may come under reverse-bias. For avoiding thermal destruction of the module, the reverse current at a reverse-bias of $12-15 \mathrm{~V}$ should not exceed a certain limit. Theoretically, silicon solar cells should stand a reverse-bias above $50 \mathrm{~V}$ without breaking down. Especially in mc cells, however, pre-breakdown appears already from 5 to $10 \mathrm{~V}$ reverse-bias. Therefore, the investigation of the pre-breakown mechanism in mc solar cells is of considerable practical importance.

A very successful method for locally studying such breakdown phenomena is LIT (Breitenstein and Langenkamp 2003). This technique images local power dissipation by imaging with an infrared (IR) thermocamera at $3-5 \mu \mathrm{m}$ wavelength, whereby the lateral heat diffusion is suppressed to a certain degree by the dynamic (pulsed bias) operation mode. With pulsed reverse-bias in the dark, LIT allows to investigate both IFE-type and avalanche-type breakdowns. With pulsed homogeneous light illumination under constant reverse-bias, LIT also allows to image the local avalanche multiplication factor (to be published in Progress in Photovoltaics). However, the spatial resolution of LIT is limited because of the inevitable lateral blurring owing to thermal diffusion. Electroluminescence (EL) imaging under reverse-bias, which does not suffer from thermal blurring, is an alternative technique to study pre-breakdown phenomena (Gaylord 1966). In this contribution we report the first attempts to use EBIC under high reverse-bias to study local avalanche phenomena in solar cells with high spatial resolution. One practical problem for this investigation is that, at high reverse-bias where avalanche multiplication is expected, there is already considerable pre-breakdown activity in the sample, leading to a reverse current of tens of milliamperes with a large noise contribution. Usual EBIC current amplifiers do not allow the compensation of such a high d.c. current. Therefore, we have used a simple d.c.-separation circuit between the sample and the current amplifier, which is sketched in Figure 8. A similar circuit was already used by Heydenreich 

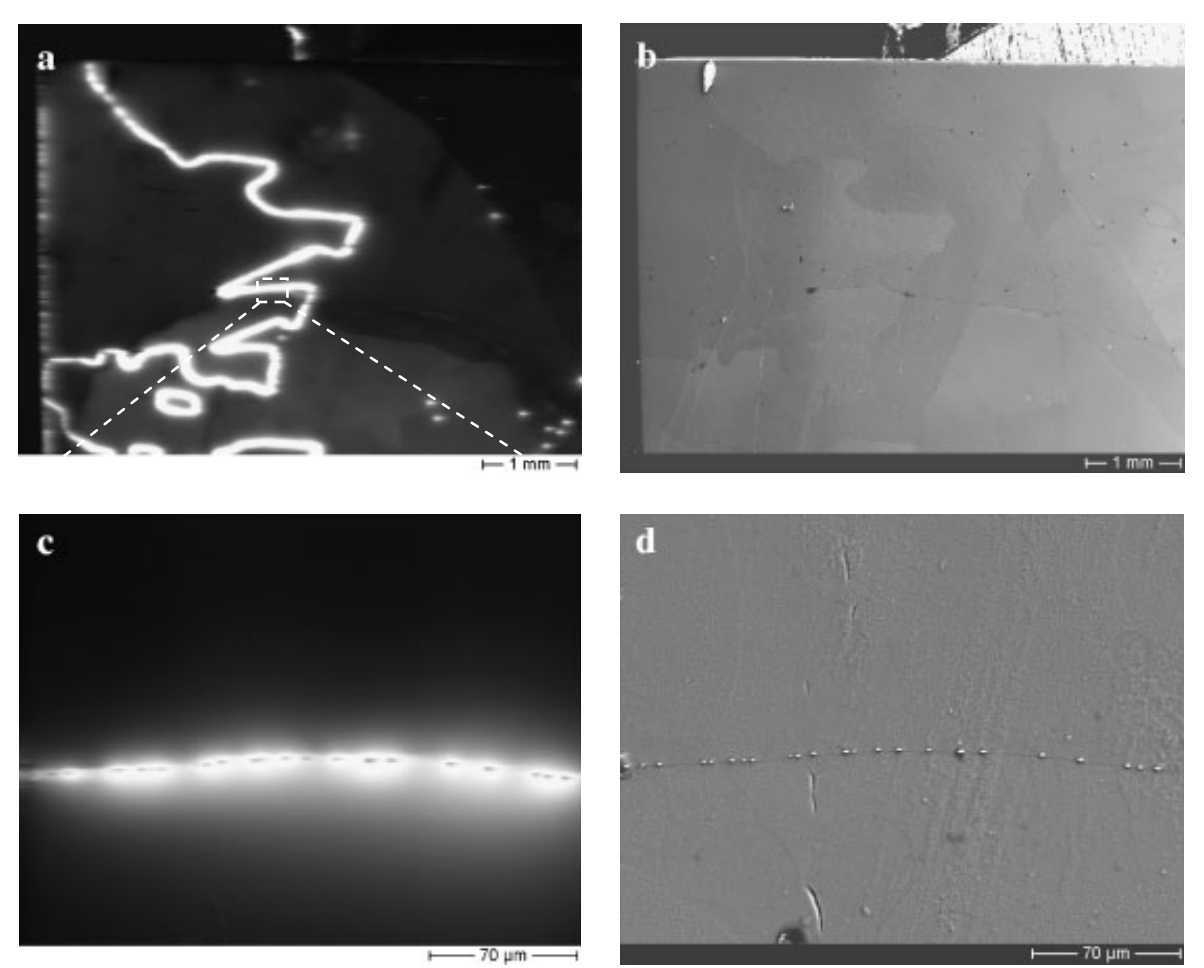

Fig 7. Low magnification (a, b) and high magnification (c, d) backside EBIC images (a, c) and SE images (b, d) of the lightly etched backside of a solar cell with conductive channels caused by $\mathrm{SiC}$ precipitates.

et al. (1981). Resistance $R_{1}$ serves as a current stabilization resistance for the sample current, capacitor $\mathrm{C}$ blocks the d.c. current, and resistor $R_{2}$ converts the following current amplifier into a voltage amplifier. In fact, $R_{1}$ is the dominant electric load for the EBIC, as long as the differential resistance of the sample is below $R_{1}$.

Figure 9 shows the results of these different methods for imaging pre-breakdown phenomena in a piece of a solar cell, which is investigated in all cases at a reverse-bias of $14 \mathrm{~V}$. Figure 9(a) shows the LIT image measured with pulsed reverse-bias in the dark, which shows the distribution of the reverse current density. The horizontal dark lines are the grid lines of the cell and the vertical dark stripe at the right is a contact stripe. Figure 9(b) was measured with constant reverse-bias by homogeneously illuminating the sample with pulsed light. In this operation mode the signal is proportional to the local avalanche multiplication factor, and any breakdown currents, which are not influenced by the pulsed light, are suppressed by the lock-in technique (Breitenstein and Langenkamp 2003). Again, the noisy region at the right is the contact stripe. At $13 \mathrm{~V}$ reverse-bias, this image looked very homogeneous, in contrast to Figure 9(b) measured at $14 \mathrm{~V}$. Hence avalanche effects are starting only above $13 \mathrm{~V}$ in this sample. Comparing (a) and (b) shows that there are both similarities and dissimilarities. Hence, obviously the breakdown mechan-

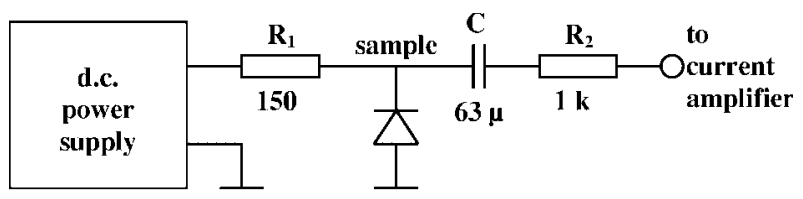

Fig 8. Separation circuit for EBIC investigation of local avalanche currents in the presence of strong pre-breakdown current of the sample.

ism is not only avalanche-type, but also IFE-type. Figure 9(c) shows a reverse-bias EL image of this region. Here the correlation to (a) is very clear, but the spatial resolution is clearly better than for the LIT images. Obviously, the pre-breakdown sites are basically single spots, which just cannot be resolved in the LIT images. For studying the avalanche process with higher spatial resolution, EBIC images have been taken at $14 \mathrm{~V}$ reverse-bias in the region indicated in Figure 9(a) and (b) using the circuit shown in Figure 8. Again, up to $13 \mathrm{~V}$ reverse-bias the EBIC image looked very homogeneous. Only above $13 \mathrm{~V}$, and only by using an acceleration voltage above $30 \mathrm{kV}$ and the maximum possible beam current, some inhomogeneities appeared. The EBIC image Figure 9(d), which was measured in the region indicated in (a) to (c), shows a number of spots (microplasma) and bright lines, which obviously coincide with grain boundaries. The darkbright contrasts at the upper edge and at the 

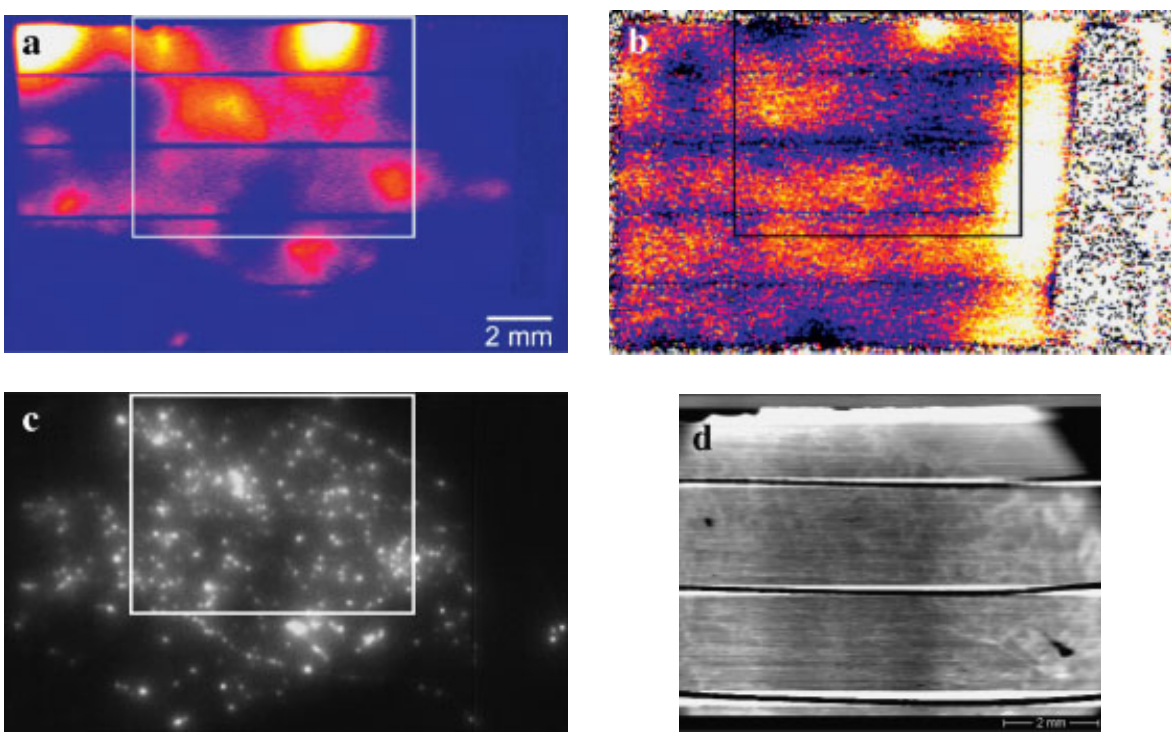

Fig 9. Piece of a multicrystalline Si solar cell at a reverse-bias of $14 \mathrm{~V}$ : (a) dark LIT image of the pre-breakdown current distribution, (b) illuminated LIT image of the avalanche multiplication factor distribution, (c) EL image and (d) EBIC image of the region indicated in $(\mathrm{a}-\mathrm{c})$ using the circuit of Figure 8.

metallic grid lines of the sample are because of a residual differentiating characteristic of the d.c.-separation circuit of Figure 8. Obviously the avalanche multiplication is largest close to the grain boundaries. There is only a weak correlation visible to Figure 9(b), which was actually expected. May be the use of the circuit of Figure 8 and the very high beam current has corrupted the quantitative result to a certain degree. In future we hope to solve this problem by using lock-in EBIC. However, we see some correlations between the EL image (c) and the EBIC image (d). So the groups of bright spots in the right part of the indicated region in (c) correspond to groups of lines and spots in (d), and in both images the top right region is free of defects. At least, it could be shown here that grain boundaries and other breakdown sites may generate microplasma (avalanche multiplication sites) under sufficiently high reverse-bias, which can be studied by EBIC.

\section{Discussion}

The temperature-dependent EBIC and PL measurements have shown that "anomalous" temperature behavior of BB luminescence and type-2 $c(T)$ behavior in EBIC have the same physical origin. Further work is needed to get also quantitative data from luminescence, i.e. to extract the contamination level $N_{\mathrm{M}}$ from the temperature behavior of the efficiency/intensity of the BB line. Then a detailed analysis of the defect contamination can be realized in a fully nondestructive manner by using the temperature dependence of the BB light.

The backside EBIC investigations have shown that this unusual geometry allows the reliable detection of conductive channels crossing the whole solar cell. These channels are caused by nitrogendoped (n-type) cubic SiC filaments, which are formed already in the block-casting process of the material. It should be mentioned that, according to our experiences, all material-induced ohmic shunts in mc solar cells are caused by these filaments, but not all filaments are causing shunts. Obviously these channels are not always in ohmic contact to the metallic back contact of the cells. It still has to be investigated under which conditions the $\mathrm{SiC}$ filaments are causing shunts.

In addition to LIT and reverse-bias EL imaging, EBIC may also be helpful for the physical investigation of pre-breakdown phenomena in mc solar cells. By using a simple d.c.-separation circuit, microplasma formation (local avalanche multiplication) could be investigated in spite of the presence of strong leakage currents. While basically bright spots appear in the EL images, the EBIC microplasma imaging shows bright lines, which are obviously grain boundaries. Note that avalanche multiplication needs a high electric field and a large free path length of the carriers. So the existence of microplasma at grain boundaries might be an indication of the gettering activity of these boundaries. Owing to this gettering activity the silicon material close to the grain boundaries is especially clean, which increases the probability of avalanche multiplication there. 


\section{Acknowledgements}

This work was supported by the BMU project $0327650 \mathrm{~B}+\mathrm{D}$ (SolarFocus).

\section{References}

Bauer J, Breitenstein O, Lotnyk A, Blumtritt H: Investigations on different types of filaments in multi-crystalline silicon for solar cells. Proceedings of the 22nd European Photovoltaic Solar Energy Conference and Exhibition, September, Milan, Italy, 994-997 (2007a).

Bauer J, Breitenstein O, Rakotoniaina JP: Electronic activity of $\mathrm{SiC}$ precipitates in multicrystalline solar silicon. Phys Stat Sol (a) 204(7), 2190-2195 (2007b).

Breitenstein O, Langenkamp M: Lock-in ThermographyBasics and Use for Functional Diagnostics of Electronic Components. Springer, Berlin, Heidelberg, New York (2003).

Gaylord JW: Microplasma observations in silicon junctions using a scanning electron beam. J Electrochem Soc 113, 753 (1966).
Heydenreich J, Blumtritt H, Gleichmann R, Johansen H: Combined application of SEM(EBIC) and TEM for the investigation of the electrical activity of crystal defects in silicon. Scanning Electron Microscopy I, SEM Inc., AMF O'Hare, Chicago, 351-365 (1981).

Holt DB, Joy DC (Eds.): SEM Microcharacterization of Semiconductors. Academic Press, London (1989).

Holt DB: The role of defects in semiconductor materials and devices. Scanning Microsc 10(4), 1047-1078 (1996).

Kittler M, Arguirov T, Fischer A, Seifert W: Silicon-based light emission after ion implantation. Opt Mater 27, 967-972 (2005).

Kittler M, Seifert W: Two classes of defect recombination behaviour in silicon as studied by SEM-EBIC. Scanning 15, 316-321 (1993).

Kittler M, Ulhaq-Bouillet C, Higgs V: Influence of copper contamination on recombination activity of misfit dislocations in $\mathrm{Si} / \mathrm{SiGe}$ epilayers: temperature dependence of activity as a marker characterizing the contamination level. J Appl Phys 78, 4573-4583 (1995).

Kveder V, Kittler M, Schröter W: Recombination activity of contaminated dislocations in silicon: a model describing the electron-beam-induced current contrast behaviour. Phys Rev B 63, 115208, 1-11 (2001). 